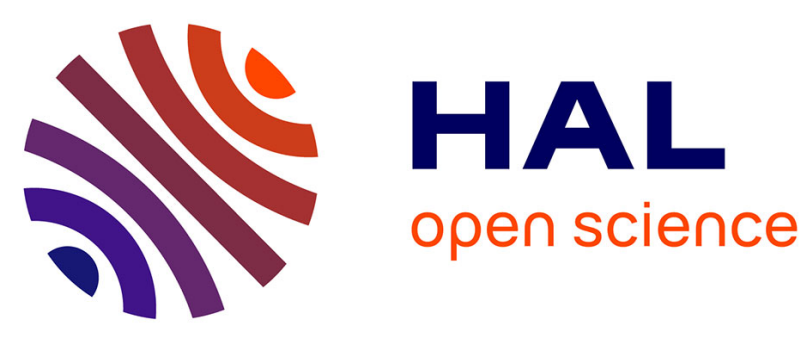

\title{
Que faire des différences ?
}

Olivier Rey

\section{- To cite this version:}

Olivier Rey. Que faire des différences ?. Recherches de science religieuse, 2013, 101 (3), pp.329-350. hal-00935010

\section{HAL Id: hal-00935010 https://hal.science/hal-00935010}

Submitted on 22 Jan 2014

HAL is a multi-disciplinary open access archive for the deposit and dissemination of scientific research documents, whether they are published or not. The documents may come from teaching and research institutions in France or abroad, or from public or private research centers.
L'archive ouverte pluridisciplinaire HAL, est destinée au dépôt et à la diffusion de documents scientifiques de niveau recherche, publiés ou non, émanant des établissements d'enseignement et de recherche français ou étrangers, des laboratoires publics ou privés. 
Recherches de science religieuse, tome $101, \mathrm{n}^{\circ} 3$, automne 2013, p. 329-350.

\title{
Que faire des différences?
}

\author{
Olivier REY
}

\section{Altérité et différence}

Il y a des mots qu'il est assez vain de vouloir définir, dans la mesure où la définition, dans son énoncé même, suppose implicitement que l'on saisisse déjà le sens du terme qu'on prétend expliciter. Il en va ainsi avec le mot « autre ». « Autre », dit le dictionnaire, " permet au sens le plus général de distinguer, de différencier, par rapport à une première partie donnée ou connue servant de point de référence, une ou plusieurs personnes, un ou plusieurs éléments à l'intérieur d'une seconde partie ${ }^{1} »$. Cependant, comment pourrait-on distinguer, différencier, séparer une première partie d'une seconde sans savoir déjà ce que c'est que d'être «autre »? L'altérité, le caractère de ce qui est autre, relève de ces notions fondamentales nécessaires à l'exercice de la pensée, et dont le sens ne peut être qu'obscurci par les efforts pour l'éclaircir. Si quelque Nathanaël s'obstinait, malgré tout, à réclamer des explications, on lui conseillerait de déchirer ces pages, de jeter ses livres et de sortir de chez lui : nul doute qu'il n'y revienne en sachant ce que signifie le mot « autre».

Le terme de «différence » est apparenté à celui d'altérité mais appelle, quant à lui, quelques précisions. Le dictionnaire définit la différence comme «caractère ou ensemble de caractères qui dans une comparaison, un ordre, distinguent un être ou une chose d'un autre être, d'une autre chose ». La différence suppose, d'évidence, l'altérité des éléments entre lesquels elle est établie ou constatée ; elle suppose, également, une comparaison ou un ordre entre ces éléments. Or on ne compare jamais, on n'établit jamais aucun ordre entre deux entités radicalement étrangères l'une à l'autre : dès lors, la différence est une distinction qui s'opère à partir d'un fond commun. Par exemple, un chêne diffère d'un hêtre à l'intérieur de la catégorie commune des arbres. Dire qu'un chêne diffère d'une montagne devient bizarre, car on perçoit mal ce qu'il y a de commun entre un chêne et une montagne, hors la qualité minimale de «chose ». Enfin, bien que l'assertion ne soit pas absolument fausse, on craindra pour la santé mentale de qui affirme qu'un chêne et un match de football sont différents, car un esprit sain ne compare que des choses comparables.

On doit noter que l'altérité ne préjuge pas de la présence ou de l'absence de traits communs entre les termes qu'elle distingue. Ces traits communs entre deux choses peuvent être si développés qu'on ne repère entre elles aucune différence, sinon leur situation dans l'espace. Ainsi, par exemple, deux pièces de monnaie qui sortent de la fonderie. Leibniz a

\footnotetext{
${ }^{1}$ Trésor de la Langue Française, CNRS/Gallimard, 16 vol., 1971-1994.
} 
contesté qu'il existât au monde deux entités absolument identiques : d'après lui, une analyse suffisamment fine doit toujours faire apparaître une différence, si petite soit-elle. De fait, dans l'exemple que nous avons pris, une analyse microscopique permettrait sûrement de repérer quelque infime différence entre les deux pièces $^{2}$. Reste que ce n'est pas cette différence qui nous fait parler d'une pièce, et d'une autre pièce - différence qui nous est tout à fait indifférente, et ne doit surtout pas être prise en compte pour que la monnaie puisse jouer son rôle. Pour nous, une pièce est autre qu'une pièce de même valeur non parce qu'elle serait différente de celle-ci, mais parce qu'au lieu d'une pièce il y en a deux. La différence, si elle existe, est neutralisée. À l'inverse, deux choses peuvent être si radicalement autres qu'on ne saurait les dire différentes, tant fait défaut le terrain commun qui permettrait d'apprécier leurs différences. Autrement dit, il peut y avoir de l'altérité là où on ne décèle aucune différence, comme là où la disparate est si grande qu'elle excède toute différence.

Les notions générales d'altérité et de différence ont naturellement cours dans le domaine humain. Non seulement elles y ont cours, mais le domaine humain n'est pas un de leur champ d'application parmi d'autres, il est le lieu primordial où elles prennent sens. On retrouve d'ailleurs, entre les hommes, toute l'étendue sémantique de l'altérité : en même temps que l'autre être humain est mon semblable, sur fond de cette similitude essentielle se perçoivent d'innombrables différences, de tous ordres. Apparaît même, entre les intériorités, une forme d'incommensurabilité. Il y a entre les humains une altérité qui est à la fois en deçà de la différence (la simple distinction des êtres sur fond de leur similitude, qui permet, par exemple, de les compter), et au-delà (la présence d'un incommensurable au sein du commensurable, une différence qui échappe à toute mesure).

\section{La différence entre l'humain et le non-humain}

Il existe pour les êtres humains deux différences particulièrement structurantes : d'une part une différence externe, entre l'humain et le non-humain, d'autre part une différence interne, entre les hommes et les femmes. L'humain ne se conçoit lui-même qu'en se distinguant de ce qu'on a coutume d'appeler, en Occident, la nature et le divin (distinctions qui n'ont de sens, rappelons-le, qu'en fonction d'une certaine parenté entre les termes: l'homme est, de quelque manière, apparenté à la nature comme aux dieux). Il se peut que des anthropologues trouvent à redire à une telle formulation, exhibant des cultures où les hommes vivent en si étroite communion avec la nature et les dieux que nos classifications se révèleront inopérantes. Dans le totémisme australien, par exemple, chaque clan se conçoit comme descendant d'un animal (voire d'une plante) - le totem. Reste que les membres du clan de l'opossum ne se comportent pas entre eux, ou avec les membres du clan du kangourou, de la même manière qu'avec un opossum ou un kangourou : la différence entre humains et animaux ne leur est donc pas inconnue, elle leur est même très sensible. Quoi qu'il en soit, la

\footnotetext{
${ }^{2}$ La physique contemporaine, cependant, n'est pas leibnizienne, en ce qu'elle postule que les particules élémentaires sont rigoureusement indiscernables. Cette indiscernabilité ne relève pas des limites de nos moyens d'investigation, elle est de principe dans la théorie.
} 
discussion avec un relativiste culturel de stricte obédience étant à la fois épuisante et stérile (ne serait-ce que parce que ce type de relativisme culturel, si prompt à dénoncer les illusions occidentales, est lui-même un pur produit occidental), à essayer de l'amadouer nous préférons confesser tout net notre provincialisme intellectuel : nous penserons avec les catégories que la tradition occidentale nous a léguées.

Au sein de cette tradition, l'humain est nettement distingué du reste de la nature. Selon Freud, la science moderne aurait infligé à l'homme trois grandes blessures narcissiques : le système de Copernic, qui l'a exilé du centre de l'univers, le darwinisme, qui l'a placé dans la continuité des animaux, et la psychanalyse, qui lui a montré que, du fait de l'inconscient, il n'est pas maître chez lui. En vérité, deux de ces blessures n'en sont pas : dans le système de Ptolémée le centre n'avait rien d'un lieu privilégié, il était le lieu le plus vil du cosmos, et le système de Copernic, qui plaçait la terre «au-dessus » du soleil, fut en son temps critiqué comme un produit délirant de la vanité humaine ; quant à la révélation de l'inconscient, peutêtre a-t-elle choqué une certaine conscience bourgeoise qui se voulait maîtresse d'elle-même comme les propriétaires l'étaient de leurs richesses, mais saint Paul n'avait pas attendu la psychanalyse pour remarquer qu'il ne faisait pas toujours le bien qu'il voulait, et qu'il lui arrivait de faire le mal qu'il ne voulait pas. Plus généralement, les chrétiens ont toujours su qu'ils ont besoin d'une grâce qui ne dépend pas d'eux. Reste le darwinisme qui, lui, a bel et bien été une blessure narcissique, qui continue de faire sentir ses effets : on ne se remet pas facilement de l'estompement d'une frontière qui paraissait essentielle à l'humanité. Dans un premier temps, la science moderne a durci la séparation entre l'homme et l'animal, en tendant à ne plus considérer le second que comme un automate (quand bien même les mots « animal » et « âme » ont la même racine) ; dans un second temps, elle a considéré l'homme comme un animal parmi d'autres: on conçoit que la conjonction des deux mouvements ait semé le trouble. En fait, l'essentiel de la difficulté tient à l'ambiguïté du mot «homme », qui renvoie aussi bien aux êtres parmi lesquels nous accédons à l'humanité, avec qui nous entrons en relation et parlons (des semblables qui, en tant que tels, sont impossibles à objectiver), qu'à l'espèce homo sapiens, produit tardif de l'évolution et objet de science. Cette ambiguïté du vocabulaire n'est pas à déplorer : elle reflète l'ambiguïté de notre condition, spirituelle et terreuse. Mais elle est aussi lourde de confusions, à chaque fois que l'un des sens du mot prétend régenter l'autre - que ce soit au mépris de la science (comme si les enseignements de celle-ci étaient congédiables à volonté), ou au mépris de notre expérience immédiate (quand nous parlons à quelqu'un, nous parlons à autrui, non à un produit de l'évolution).

Aux temps où l'homme vivait en contact direct avec la nature, on comprend qu'il se soit d'abord différencié par rapport à des éléments de cette même nature, en premier lieu les animaux. Aujourd'hui cependant, en nos pays, pour beaucoup d'enfants la première souris rencontrée est la souris d'ordinateur, non le petit rongeur à museau pointu courant sur ses quatre pattes agiles : la métaphore s'est inversée. Autrement dit, le premier autre-qu'humain tend à devenir non plus l'animal, mais la machine. Dès lors, la blessure narcissique ne consiste plus tant, pour l'homme, à être ravalé au rang de l'animal, qu'à être comparé à une machine - et, qui plus est, à révéler dans cette comparaison toute l'étendue de ses 
insuffisances. Quiconque, par exemple, a fréquenté un hôpital moderne a pu constater à quel point le corps humain paraît fragile et archaïque en regard de l'appareillage high-tech qui l'environne et pallie ses déficiences. Les êtres humains sont pris, face à ces machines, de ce que Günther Anders a appelé la «honte prométhéenne » (ou le déshonneur) : le sentiment mortifiant de ne pas être à la hauteur de ce qui est, pourtant, une production humaine, l'impression d'être jugé par cette production et déclaré inapte ${ }^{3}$. C'est ainsi que peut s'expliquer, en réaction, une certaine valorisation contemporaine du pulsionnel, contre la raison qui jadis faisait la gloire de l'homme par rapport à l'animal : un essai de fuite devant l'humiliation infligée par la machine. Pourtant une machine, quelle qu'elle soit, n'exerce sa fascination qu'à travers une confrontation injuste pour les facultés humaines, puisque toujours limitée au terrain extrêmement étroit où cette machine excelle et en dehors duquel elle est sans ressources. L'ordinateur qui bat les plus grands maîtres aux échecs ne sait rien faire d'autre que de jouer aux échecs. Alan Turing, en 1950, a imaginé un test dont il estimait que, s'il était réussi par une machine, celle-ci devrait être reconnue comme pensante; il pensait aussi que cinquante ans plus tard, ce pas serait franchi. Il se trouve que, malgré les progrès spectaculaires accomplis par l'informatique durant le dernier demi-siècle, personne ne se vante plus de fabriquer un ordinateur capable de passer le test. Le sentiment d'infériorité de l'homme par rapport à la machine, pour répandu qu'il soit, n'en repose pas moins sur une appréciation biaisée de la réalité.

\section{La différence entre l'homme et la femme}

Il vaut la peine de dire un mot sur le test imaginé par Turing, souvent évoqué mais rarement décrit. La première étape consiste en un jeu de l'imitation : un interrogateur doit deviner, à la teneur des réponses que lui donnent un homme et une femme avec lesquels il ne communique que par messages dactylographiés, qui est l'homme, qui est la femme - le but de la femme étant de lui faire découvrir la vérité, celui de l'homme de l'induire en erreur. On enregistre le taux d'échec de l'interrogateur à ce jeu. Dans un second temps, à l'insu de l'interrogateur, une machine est substituée à l'homme, avec la même mission que lui, à savoir se faire passer pour la femme. Si le taux d'échec de l'interrogateur est comparable ou supérieur à ce qu'il était précédemment, on doit, selon Turing, convenir que la machine pense. On dit souvent que le test de Turing met à l'épreuve la faculté d'une machine à passer pour un être humain. Mais en ce cas, le test serait déséquilibré : la machine devrait passer pour ce qu'elle n'est pas - un humain -, en comparaison d'un humain devant simplement passer pour ce qu'il est. D'où, pour remédier à ce défaut, les deux étapes du test, qui permettent de comparer les facultés respectives d'un homme et d'une machine à passer pour une femme, ce que ni l'un ni l'autre ne sont. Un point est à souligner : ce fameux test met sur le même plan d'une part la différence entre une machine et une femme, d'autre part la différence entre un

\footnotetext{
${ }^{3}$ Voir Günther Anders, L'Obsolescence de l'homme - Sur l'âme à l'époque de la deuxième révolution industrielle [1956], trad. Christophe David, Éditions de l'Encyclopédie des nuisances / Ivrea, 2002.
} 
homme et une femme. Autrement dit, avant de mettre à l'épreuve la qualité «pensante » d'une machine, il accorde à la différence sexuelle un statut essentiel.

En cela, il n'a pas tort. La différence entre hommes et femmes est en effet fondamentale, tant dans l'organisation des sociétés humaines que dans les structures psychiques individuelles. Il n'est, pour le mesurer, que de songer aux langues que nous parlons. Nous ne disposons pas de pronoms différents pour désigner les grands ou les petits, les jeunes ou les vieux, les proches et les étrangers, nos amis ou nos ennemis. Pour évoquer une personne nous n'avons que deux pronoms : «il», « elle». Le masculin, le féminin. Il en résulte - surtout si l'on tient compte du fait que le sujet humain se constitue dans le langage, est un « parlêtre »-que la différence des sexes n'est pas une différence parmi d'autre, mais la différence à partir de laquelle les autres différences peuvent être pensées. Bien sûr, on peut disserter sur la différence de façon théorique, abstraite, avec de multiples références à la tradition philosophique depuis Aristote jusqu'à l'école analytique - après quoi on illustrera d'exemples les notions dégagées et discutées. Le côté un peu vain de la démarche est que le lecteur sera sans cesse obligé, pour saisir le sens du texte, de redonner aux concepts la chair que le rédacteur aura tout fait pour leur retirer. Ici, nous laisserons la chair sur les os, avec le sentiment qu'en s'attardant sur la différence entre hommes et femmes nous ne renonçons pas au général pour nous complaire dans le particulier, mais nous plaçons sur le terrain où le général trouve ses fondations. Remarquons, au passage, que la pensée mythique reconnait à la différence sexuelle une telle importance, que cette différence se révèle plus infranchissable que la barrière des espèces. Ainsi, dans le Brihadaranyaka Upanishad, on voit les espèces engendrées successivement par la fuite de la femelle devant le mâle : la femme, pour se cacher de l'homme, se transforme en vache; alors l'homme, pour la rejoindre, se transforme en taureau ; la vache, pour se cacher du taureau, devient jument mais le taureau, pour s'unir à nouveau à elle, devient étalon, etc. Dans les mythes grecs les dieux antiques, si prompts aux métamorphoses, ne franchissaient jamais la frontière entre les sexes : Zeus pouvait prendre la forme d'un taureau, d'un aigle ou d'un cygne, par celle d'une femme ${ }^{4}$. La vérité est que la différence homme/femme, avant d'être un cas particulier de la différence sexuelle, en a d'abord été le modèle, qui s'est ensuite projeté sur le monde, bien au-delà, du reste, de ce qu'on classifierait aujourd'hui comme mâle ou femelle. Selon Durkheim et Mauss, «les premières catégories logiques ont été des catégories sociales ${ }^{5} »:$ le fait que dans une langue comme le français (mais elle est loin d'être une exception), tous les substantifs soient ou masculins, ou féminins, est la marque du rôle primordial qu'a joué le partage hommes/femmes dans la constitution des sociétés.

\footnotetext{
${ }^{4}$ Le cas de Tirésias, qui fut changé en femme et qui, sept ans plus tard, retrouva son sexe d'origine, est si isolé qu'il est l'exception qui confirme la règle.

${ }^{5}$ Émile Durkheim et Marcel Mauss, « De quelques formes primitives de classification, contribution à l'étude des représentations collectives », in L'Année sociologique, 1903, repris dans Marcel Mauss, Essais de sociologie, Minuit, 1968, p. 225.
} 
Ce partage, reconnu et institué, permet aux individus de vivre ensemble. En effet, c'est à partir de la part d'opacité que comporte chaque sexe pour l'autre qu'il est permis à un être humain d'admettre la part d'opacité que comporte chaque autre être humain. C'est à partir de la différence des sexes que nous pouvons faire place à la différence, et comprendre que la différence n'est pas une objection à la vie en commun. Sans cet ancrage, toute différence deviendrait une occasion de scandale. On ne voudrait plus être qu'avec ceux qui nous ressemblent le plus, et les phénomènes de ségrégation ne feraient que se multiplier. Il n'est que trop évident que la différence entre hommes et femmes ne conjure pas les attitudes hostiles vis-à-vis de la différence, ni les conduites ségrégationnistes. Elle n'en demeure pas moins un point essentiel à partir duquel l'ouverture à l'altérité peut se faire.

Déjà dans l'Éden, c'est autour de cette question que les choses se jouent. Une certaine tradition veut que ce soit dans l'infinitude de sa volonté que l'être humain trouve la marque qu'il a été fait à l'image du Dieu infini. Mais même en admettant ce point, cette image n'est ressemblante au Créateur qu'en tant que la volonté sait mettre des bornes à ce qu'elle convoite - comme Dieu a mis une limite à l'exercice de sa puissance afin qu'advienne un monde où vivent des êtres libres (la suprême puissance n'est pas celle qui s'annexe tout, mais celle qui sait se maîtriser elle-même pour être avec d'autres). De là le fameux arbre défendu, dit arbre à connaître le bien et le mal. Les langues archaïques n'ayant pas de termes pour nommer les totalités, elles désignaient celles-ci pas leurs extrémités : on ne disait pas le monde mais le ciel et la terre ; et s'il avait existé un arbre à connaître le ciel et la terre, la connaissance en question n'aurait pas consisté à distinguer le ciel de la terre, mais à tout savoir de la nature. De même, pour évoquer l'ensemble de la connaissance morale on disait : connaître le bien et le mal ${ }^{6}$. Il est probable que les fruits de l'arbre défendu n'avaient aucune vertu particulière. La seule chose qui distinguait l'arbre, c'était l'interdit qui le touchait. C'est en respectant cet interdit, c'est-à-dire en acceptant que quelque chose, dans le monde, ne soit pas à sa libre disposition, que l'être humain ressemble à Dieu parce que c'est ainsi que, comme Dieu, il est relation. De là le passage du singulier au pluriel quand Elohim dit dans la Genèse $(1,27)$ : «Nous ferons Adam à notre image, selon notre ressemblance. Elohim crée l'Adam à son image, à l'image d'Elohim il le crée, mâle et femelle il les crée. » Ce passage signifie que ce n'est pas l'individu isolé qui est à l'image de Dieu, que c'est au contraire en renonçant au fantasme d'être tout solitairement - fantasme que viendra flatter le serpent -, c'est en étant avec les autres, et l'autre de l'autre sexe, que l'être humain a part à la totalité divine. Si la relation entre l'homme et la femme peut sembler un cas particulier de la relation de l'être humain à son prochain c'est, en même temps, ce cas particulier qui est le gardien du cas général.

\footnotetext{
${ }^{6}$ Dans le livre de Samuel la femme de Teqoa s'adresse en ces termes à David : «Monseigneur le roi est comme l'Ange de Dieu pour saisir le bien et le mal» (2 S 14.17); trois versets plus loin on lit : «Monseigneur a la sagesse de l'Ange de Dieu, il sait tout ce qui se passe sur la terre » (2 S 14.20) - un terme totalisant a ici fait son apparition, qui se substitue à « saisir le bien et le mal ».
} 
Le péché a abîmé cette relation. À partir du moment où chacun a prétendu au tout, sans l'autre, les hommes et les femmes sont entrés les uns envers les autres dans un régime de convoitise et de domination. Mais ce régime n'est pas inéluctable. Ce qu'annonce Paul dans l'épître aux Galates $(3,28)$ - « il n'y a plus ni Juif ni Grec, il n'y a plus ni esclave ni homme libre, il n'y a plus l'homme et la femme, car tous vous ne faites qu'un dans le Christ Jésus $^{7} »-$, ce n'est pas l'abolition de la différence sexuelle, mais le fait que cette différence n'implique plus convoitise et domination. Il n'y a plus l'homme et la femme en concurrence l'un avec l'autre, du fait de leur différence, mais une différence entre l'homme et la femme qui est pour la relation et pour l'unité . $^{\text {. }}$

Il a fallu attendre l'époque moderne pour que l'importance fondamentale et structurante de la différence des sexes pour les sociétés humaines et la pensée se trouve remise en cause. Ce fut d'abord le passage progressif, amorcé au XVIII ${ }^{\mathrm{e}}$ siècle, d'un monde peuplé d'hommes et de femmes à un monde peuplé d'êtres humains, secondairement masculins ou féminins. Une telle mutation est lourde de conséquences. Car lorsque l'humanité n'est pas ce qui rassemble les hommes et les femmes, mais ce qui précède et transcende le caractère masculin et féminin, alors il devient possible de considérer ce caractère sexué comme subsidiaire, et lui refuser tout rôle dans la vie sociale. «Le monde qui s'organise selon la différence des sexes est celui dont nous ne voulons plus ${ }^{9} »$, déclare Élisabeth Badinter, dont nous ne citons les propos que dans la mesure où, loin de traduire un point de vue isolé, ils expriment au contraire une façon de penser très répandue. Mais alors, une question de fond mériterait d'être posée. Il fut un temps où certains marxistes se demandaient si dans une société communiste accomplie les anciennes langues pouvaient demeurer en usage, ou s'il allait falloir inventer une nouvelle langue,

\footnotetext{
${ }^{7}$ La traduction est souvent fautive qui écrit : «il n'y a plus ni homme ni femme » (c'est le cas dans la Bible de Jérusalem). Cette traduction efface le changement de construction, forcément significatif, dans le texte grec,

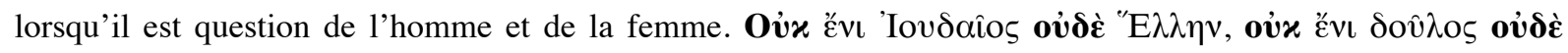

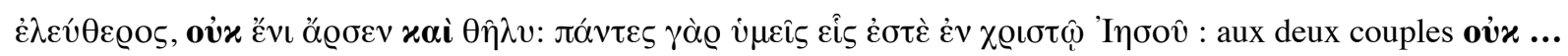
oùḋ̇..., qu'on peut traduire par «ni... ni... », lorsqu'il s'agit du Juif et du Grec, de l'esclave et de l'homme libre, succède oủx... xaì, soit une seule négation (ởx) qui s'applique à ce qui suit, c'est-à-dire à l'homme

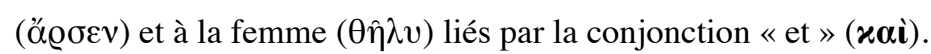

${ }^{8}$ Voir Anne-Marie Pelletier, «Il n'y a plus l'homme et la femme, Communio, nºXVIII, 2, 1993.

Il est à noter que dans le mythe grec de l'androgyne, tel qu'on le trouve exposé dans le Banquet de Platon, la séparation entre hommes et femmes provient de la section d'êtres primitivement complets qui, pour cette raison, menaçaient la prééminence des dieux; depuis lors, en s'appariant, l'homme et la femme chercheraient à retrouver quelque chose de leur unité primitive. Dans le récit biblique, il en va autrement : la sexuation n'est pas une punition mais, au contraire, un don de Dieu, pour que l'humain ne soit pas seul. L'unité de l'homme et de la femme n'est pas un état initial qu'il s'agit de retrouver, elle ne résulte pas davantage d'une complémentarité prédéfinie - elle est, essentiellement, une promesse et une visée. La complémentarité est une différence donnée, en quelque sorte, avec son mode d'emploi, sans au-delà, qui se referme sur elle-même sur le mode de l'emboîtement. Or l'autre sexe n'est pas complémentaire, il est une aide à l'accomplissement de l'humanité en chacun, dans la relation à l'autre.

${ }^{9}$ Paroles conclusives prononcées, «d'une voix grave et insistante», à la tribune du $19^{\mathrm{e}}$ Forum Le Monde Le Mans qui s'est tenu du 16 au 18 novembre 2007 sur le thème « Masculin-féminin, les nouvelles frontières ».
} 
accordée aux nouveaux rapports de production. Au soulagement du plus grand nombre, Staline jugea que la langue n'était pas une superstructure de l'économie, et que les Soviétiques continueraient donc à parler leurs langues maternelles. Cependant, si l'enjeu n'est plus la collectivisation des moyens de production, mais l'abolition du monde organisé selon la différence des sexes, est-il concevable de persister à employer des langues elles-mêmes structurées selon cette différence ? Aujourd'hui, à fin d'égalité, on s'emploie à féminiser des mots qui jusque-là n'existaient qu'au masculin (écrivaine, professeure, défenseuse etc.). Mais ce procédé, en fin de compte, ne fait que renforcer encore la sexuation de la langue là où, par ailleurs, on voudrait que la différence des sexes ne compte pour rien dans l'organisation sociale $^{10}$. À quoi l'on voit que c'est bien un changement radical de langue qui serait nécessaire; une liquidation si complète de nos héritages que la volonté d'en finir avec le monde organisé selon la différence des sexes révèle son essence nihiliste.

\section{Le contexte général}

Déceler la dimension nihiliste d'un projet ne suffit pas : encore faut-il comprendre les motifs qui le nourrissent. L'un d'entre eux est facile à saisir : il réside dans l'association de différence à différend. Parce que les différences sont susceptibles d'initier ou d'alimenter des conflits, tout n'irait-il pas mieux sans elles ? Si le monde des différences a été si souvent en guerre, un monde sans différences ne serait-il pas en paix ? Il n'y a pas d'idée plus erronée. La menace la plus redoutable qui pèse sur les sociétés humaines, en effet, est l'anomie, la guerre de tous contre tous. Et cet état de décomposition survient à travers des phénomènes de mimétisme généralisé. René Girard n'a cessé d'insister sur ce point très simple aux implications colossales : les êtres humains s'imitent les uns les autres, y compris dans leurs désirs. Ce qui fait que lorsqu'un individu voit un autre que lui, ou plusieurs, désirer certaines choses, ces choses deviennent pour lui désirables et il entre en compétition pour les obtenir. Et plus il entre dans cette compétition, plus il renforce les autres dans la conviction que les choses qu'ils visent sont désirables. Au départ, les protagonistes pouvaient être assez différents les uns des autres. Mais plus la lutte entre eux se développe, s'intensifie, plus cette lutte symétrise les adversaires, arase entre eux les différences; et plus les adversaires sont indiscernables, plus la lutte qui les oppose se fait féroce et sans solution, en dehors de la sanction de la pure violence, ou de la réconciliation de la communauté au dépens d'une victime émissaire ${ }^{11}$. Il importe donc de bien saisir une chose : les différences - pour autant

\footnotetext{
${ }^{10}$ Le principe de «parité » présente une équivoque similaire. Promu pour combattre la sexuation de certaines fonctions ou certains métiers, qui contrevient à l'idée égalitaire voulant que le sexe n'intervienne pas dans le destin social des individus, le principe de parité ne cesse, pour s'appliquer, d'attirer l'attention sur le sexe des personnes. Par ailleurs ce principe, qui ne s'accompagne pas de quotas relatifs à d'autres différences (économiques, régionales, ethniques, religieuses etc.), reconnaît de ce fait même à la différence des sexes l'importance sans équivalent dont on voudrait, en même temps, qu'elle n'ait pas d'incidence sociale.

${ }^{11}$ Voir, en particulier, de René Girard, La Violence et le Sacré, Grasset, 1972, et Des choses cachées depuis la fondation du monde, Grasset, 1978.
} 
qu'elles soient reconnues et instituées -, en mettant un frein aux identifications réciproques, mettent également un frein aux emballements mimétiques.

On conçoit volontiers aujourd'hui les structures des sociétés anciennes, avec leurs rôles bien marqués, leurs ordres ou leurs castes plus ou moins nettement délimités, leurs assignations des individus à des places prédéterminées, comme des moyens pour les dominants de pérenniser leur domination. Cet aspect des choses est réel. Il ne doit cependant pas occulter un autre aspect: ces structures servaient aussi de protection contre le déchaînement de la violence. Considérons les douces colombes, symboles de la paix : en cage, elles se révèlent impitoyables pour un congénère trop faible pour se défendre, qui finit par mourir sous les coups de bec. C'est qu'il n'y a pas, entre elles, de mécanisme d'inhibition de la violence ; et cela, parce que dans la nature un individu de la gent ailée a toujours le loisir de s'enfuir - dans les airs, il est impossible de «coincer » un adversaire. Il n'en va pas de même sur terre. Quand les animaux d'une même espèce sont capables, dans leur milieu naturel, de se tuer entre eux, il existe des mécanismes instinctifs qui viennent limiter l'agressivité intra-spécifique. Ainsi chez les loups : au sein d'une meute, une hiérarchie entre les mâles s'établit à travers des affrontements; lorsque l'un des combattants prend l'avantage sur l'autre, celui qui a le dessous se met sur le dos et présente sa gorge, et cette posture de soumission a pour effet immédiat d'inhiber l'agressivité du vainqueur ; la préséance de celuici est reconnue, et ne pourra plus être remise en cause avant un certain temps. Les humains, comme les loups, sont capables de tuer leurs congénères. Mais il n'existe pas en eux de mécanismes instinctifs efficaces qui viennent limiter l'expression de la violence. Il faut donc que s'élaborent, au sein des sociétés humaines, de telles limites. Ce seront, par exemple, les structures qui, selon tels ou tels critères, assignent plus ou moins à chacun une place dans la société. La raison d'être de ces structures n'est pas seulement de protéger les puissants ; elle est également de limiter la compétition, de canaliser la violence.

Tout n'est pas réglé pour autant, loin s'en faut. Les structures sociales, en effet, n'ont pas l'efficacité de l'instinct. Les différences instituées ont une dimension arbitraire et sont donc toujours susceptibles d'être remises en cause. Elles peuvent engendrer des insatisfactions, des ressentiments : alors qu'elles devaient contenir la violence, elles peuvent à leur tour devenir un motif de se battre. D'où la profonde ambiguïté des différences entre les êtres humains ; d'où les débats contemporains sur la manière d'envisager les différences - sur le statut à leur donner, ou à ne pas leur donner. Est-il opportun de les promouvoir ou doit-on, au contraire, chercher à les atténuer ? Faut-il les instituer ou, au contraire, se refuser à en tenir compte socialement ? Ce débat n'est pas à prendre à la légère : il touche au fondement même des sociétés humaines. Pour qu'un tel débat puisse être fécond, il convient de le poser correctement. Et, pour ce faire, il est utile de savoir le situer au sein d'un contexte anthropologique et historique. Élargir la perspective n'est pas se détourner des questions actuelles : c'est, au contraire, se donner les moyens d'en mieux saisir les enjeux.

La pensée antique et médiévale concevait le monde comme cosmos ; c'est-à-dire, au sens propre du terme grec, comme un tout harmonieux (opposé au chaos), un bon arrangement d'éléments hétérogènes - ensemble bellement et bien ordonné dans la mesure 
où, dans un espace lui-même hétérogène, parcouru de frontières et hiérarchisé, chacun des éléments est sa place et, en étant ce qu'il est, en atteignant sa fin, concourt à l'harmonie du tout. Dans ce contexte, la raison humaine est cette faculté qui, en l'homme, lui permet d'accéder à la raison objective qui ordonne le monde. Le monde ancien regorge d'altérités en tous genres. Pour autant, la pensée n'y est pas perdue ; elle en surmonte la fragmentation par une multitude de réseaux analogiques, qui transcendent les frontières ontologiques. $\mathrm{Ne}$ donnons qu'un exemple : celui de l'astrologie qui, malgré la différence essentielle entre le monde céleste et le monde terrestre, trouve des analogies entre le mouvement des astres dans le ciel et les événements sur la terre.

$\mathrm{Au}$ sein de la pensée moderne il en va tout autrement. Dans le sillage du clivage terrestre-céleste, effacé d'un coup par le système de Copernic, toutes les anciennes divisions cosmiques ont été dissoutes, avec les notions d'ordre préétabli, de hiérarchies, de rapports analogiques et d'harmonie qui leur étaient attachées, pour laisser place à un espace uniforme, sans lieux privilégiés ni sens intrinsèque, régi de part en part par des lois identiques, s'appliquant à des objets formés de la même matière sous diverses formes, ayant tous le même statut ontologique. Les Européens ont progressivement cessé, au fur et à mesure qu'ils entraient dans la modernité, d'habiter un cosmos - espace fini, différencié, hiérarchiquement ordonné -, pour vivre dans l'univers moderne - espace infini, homogène, régi par la seule géométrie. Au sein du monde matériel n'existe plus d'altérité irréductible, seulement des différences.

La modernité est rupture avec les structures a priori du monde. Certaines structures, bien sûr, continuent d'exister - mais désormais, elles ne peuvent plus être pensées comme intrinsèques, elles ne sont que le résultat des processus dont le monde est le théâtre, tant sur le plan matériel que sur le plan humain. Les différences ne sont plus ce qui explique le monde mais, au contraire, ce qui est toujours à expliquer, sur fond d'indifférenciation originelle. Tous les phénomènes naturels sont rapportés, en dernière analyse, à des champs qui se propagent, à des interactions entre particules, à des flux d'énergie. Dans ce monde mécanisé seul l'être humain se trouve encore pourvu d'une intériorité. Sa conscience s'y trouve comme insularisée. C'est ainsi que peut se comprendre, non pas le processus d'individualisation et d'égalisation des conditions en lui-même, dont l'histoire occidentale des derniers siècles a été le théâtre (il faut se garder de l'hubris philosophique qui prétend déduire la réalité des idées), mais son caractère irrésistible, souligné par Tocqueville. En effet, à partir du moment où les consciences humaines se trouvent ainsi isolées au sein du monde, les sources ultimes de légitimité deviennent les individus et, parce qu'il n'y a rien de mondain qui permette de comparer une conscience à une autre, parce que "pour chaque âme, chaque autre âme est un arrière-monde ${ }^{12} »$, chaque individu dispose d'une égale légitimité (même si perdurent de grandes inégalités de fait, celles-ci ne s'en déploient pas moins sur fond d'une égalité de principe). La volonté prend clairement le pas sur l'entendement dans la hiérarchie des facultés, et la raison, d'objective, se fait subjective : son exercice ne consiste plus à accorder

\footnotetext{
${ }^{12}$ Friedrich Nietzsche, Ainsi parlait Zarathoustra, III [1884], « Le convalescent », § 2.
} 
l'homme au cosmos, mais à pénétrer les règles de fonctionnement de la nature pour, ensuite, ordonner convenablement les moyens en vue de fins qui se décident hors d'elle. Science et morale se disjoignent absolument: c'est la is/ought distinction de Hume, la séparation consommée entre l'être et le devoir-être, l'impuissance radicale à déduire le second du premier. Et de même que les valeurs ne sauraient être déduites des faits, de même les formes politiques perdent toute la légitimité qu'elles pouvaient trouver dans le fait de simplement être, ou dans des analogies avec un ordre naturel ou surnaturel (ainsi, l'analogie entre le rapport du suzerain à ses vassaux et celui de la tête par rapport aux membres, ou de Dieu à ses créatures). Les principes d'organisation des sociétés apparaissent comme étant le produit exclusif des hommes, de leur activité, de leurs échanges, de leurs confrontations et de leurs décisions. De ce point de vue, les différences socialement reconnues ne renvoient plus à un ordre des choses : elles sont toujours contingentes, susceptibles d'être remises en cause et « déconstruites ».

À cet égard, il est intéressant d'examiner le destin d'un mot comme «discrimination ». Ce terme savant est apparu en France au XIX siècle pour désigner l'action de distinguer, sans les connotations sociales qui pouvaient accompagner le terme de «distinction». Voici la définition que donnait Littré dans son dictionnaire : «Terme de psychologie, employé par Bain $^{13}$. Faculté de discerner, de distinguer. "Ce changement d'état [par lequel la conscience passe d'une modification à une autre], c'est la discrimination, et c'est le fondement de notre intelligence" (Ribot, Psychologie anglaise).» Aujourd'hui, le terme a perdu son sens technique pour devenir nettement péjoratif, et sert avant tout à désigner une injustice, voire un délit. Certes, ce n'est pas la faculté de discerner ou de distinguer qui se trouve en soi visée, mais le fait de changer de comportement en fonction de distinctions qui ne devraient pas entraîner de différences de traitement. Il n'en reste pas moins que le glissement de sens du mot est significatif d'une situation contemporaine qui, en même temps qu'elle demande le respect des différences (au nom de l'absence d'une autorité qui les transcenderait), a la hantise des comportements différenciés (au nom de l'exigence d'égalité).

\section{De quelques difficultés contemporaines : reconnaissance des différences et égalité}

Ce qui précède permet de mieux cerner deux types de difficultés inédites auxquelles les sociétés modernes se trouvent confrontées.

- Premièrement, le conflit entre la liberté et l'égalité.

La liberté est accordée à chacun au nom de l'égalité. Mais l'égalité risque vite de passer pour un mot vide en face des immenses inégalités de fait qui se prolongent ou s'installent. Réciproquement, l'égalité ne peut être maintenue dans les faits que par des limites mises à la liberté. Le problème d'autant plus aigu que s'estompe la distinction des ordres selon Pascal ordre des corps (matériel), ordre des esprits (intellectuel), ordre de la charité (spirituel). Les inégalités dans l'ordre matériel ou intellectuel peuvent se trouver relativisées par l'égalité

\footnotetext{
${ }^{13}$ Alexander Bain (1818-1903) est un philosophe et pédagogue écossais de l'école empiriste.
} 
dans l'ordre spirituel, à proportion de la place accordée à cet ordre spirituel au sein de la pensée commune. Quand cette place se réduit, les inégalités dans les autres ordres s'absolutisent, et deviennent d'autant plus insupportables.

- Deuxièmement, le conflit entre l'autonomie revendiquée du sujet vis-à-vis de la société, et le désir de reconnaissance qu'adresse le même sujet à la société. Il ne suffit pas, en effet, que la société nous laisse libre de faire ce que nous voulons, nous voulons aussi être reconnus par elle. Ce qui veut dire : reconnus non pas abstraitement, mais pour ce que nous sommes. D'où les revendications d'être reconnu dans sa différence. Cela couplé - il faut y insister - avec l'exigence d'un traitement égal. La pensée analogique n'avait aucune peine à reconnaître les différences ; mais elle le faisait à sa manière, en insérant ces différences dans des structures hiérarchiques. La pensée naturaliste moderne n'a pas cette ressource. D'où la question qui se pose aujourd'hui : est-il possible de concilier la reconnaissance de la différence d'une part, l'égalité d'autre part? L'anthropologue Louis Dumont pensait que non: «Si les avocats de la différence réclament pour elle à la fois l'égalité et la reconnaissance, ils réclament l'impossible ${ }^{14}$.» Une telle affirmation heurte tellement les idéaux actuels, qu'il vaut la peine de s'y arrêter, de l'examiner soigneusement.

Pour éclairer son propos, Dumont donne l'exemple de la latéralisation. La façon habituelle de concevoir, de nos jours, la différence droite-gauche, est de l'envisager comme une différence totalement neutre, sur laquelle, ensuite, les êtres humains vont projeter des valeurs. Dumont, quant à lui, renverse la perspective. «Il y a quelque chose d'exemplaire dans cette relation droite-gauche. C'est peut-être le meilleur exemple d'une relation concrète indissolublement liée par les sens à la vie humaine, de l'espèce que les sciences physiques ont négligée et que l'anthropologie pourrait bien restaurer ou réhabiliter. Je crois qu'elle nous enseigne avant tout que dire "concret" c'est dire "pénétré de valeur"15. » Dumont affirme que ce qui est premier n'est pas la différence, mais la valeur ${ }^{16}$. Les différences sont en nombre infini, mais nous ne sommes sensibles qu'à celles qui sont investies de valeur. Autrement dit, la reconnaissance d'une différence commence toujours par aller de pair avec une référence à la valeur. Et qui dit valeurs, dit hiérarchie par rapport à ces valeurs.

La première chose à éviter, à ce stade, est de céder à une vision trop simpliste des structures hiérarchiques. Clarifions quelques points.

1) Hiérarchie ne veut pas dire pouvoir.

L'exemple de la relation droite-gauche, fondée sur la latéralisation du corps, est là encore instructif. "Les modernes tendent à définir la valeur en relation avec la volonté arbitraire, le Kürwille de Tönnies, tandis que nous sommes ici dans le domaine de Naturwille,

\footnotetext{
${ }^{14}$ «La valeur chez les modernes et chez les autres », dans Essais sur l'individualisme, Seuil, coll. Points essais, 1991, p. 297.

${ }^{15}$ Ibid., p. 273. Dumont reproche à la pensée moderne d'avoir modifié la définition de l'être en en excluant la notion de valeur, qui pourtant y donne accès.

16 On peut, au moins formellement, établir un lien avec l'affirmation d'Emmanuel Levinas pour qui la philosophie première n'est pas l'ontologie (l'étude de l'être en tant qu'être, de l'essence et de l'existence), mais l'éthique.
} 
la volonté naturelle, spontanée. Strictement, le tout n'est pas préférable à ses parties, il leur est simplement supérieur. La droite est-elle supérieure à la gauche ? Elle est seulement opportune dans certaines circonstances. Si l'on insiste, ce qui est “désirable ", c'est d'agir en accord avec l'ordre des choses. Quant à la tendance moderne à confondre hiérarchie et pouvoir, qui donc prétendrait que la droite ait pouvoir sur la gauche ? Même sa prééminence est, au niveau de l'action, limitée à l'accomplissement des fonctions qui lui reviennent ${ }^{17}$. »

2) Une hiérarchie est relative à l'ensemble qui en englobe les termes.

Pour nous en tenir à l'exemple droite-gauche : « Nous séparons idées et faits des touts dans lesquels ils se rencontrent en réalité. Plutôt que de mettre en relation le niveau considéré - droite et gauche - avec le niveau supérieur, celui du corps, nous limitons notre attention à un seul niveau à la fois, nous supprimons la subordination en séparant ses éléments ${ }^{18}$. » Cette remarque de bon sens est susceptible d'éclairer bon nombre de situations. Quand, par exemple, l'autorité du maître sur ses élèves est contestée au nom du fait qu'il n'appartient pas à un être humain d'avoir autorité sur d'autres êtres humains, on oublie le niveau supérieur qui régit une salle de classe - en l'occurrence, le savoir - par rapport auquel cette relation d'autorité prend sens : c'est par rapport à ce savoir qu'il y a un maître et des élèves (rapports limités, en l'occurrence, à un certain contexte, et qui plus est, dans ce cas, temporaire comme l'autorité des adultes sur les enfants ; en un sens, le maître est maître en ce qu'il est lui-même davantage soumis à la discipline à laquelle il s'agit d'introduire les novices - faute de quoi, il n'est pas un maître mais un tyran).

3) Les hiérarchies, dans les sociétés traditionnelles, sont souvent complexes, enchevêtrées.

Dumont se réfère souvent à de tels schémas : A est supérieur à $\mathrm{B}$ mais, dans le domaine réservé à B au sein de l'ordre global, la hiérarchie s'inverse, c'est B qui est supérieur à A.

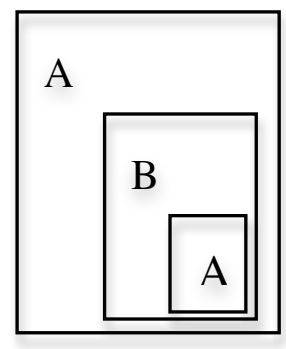

Par exemple: dans les sociétés patriarcales, l'homme domine la femme. En vertu de cette domination, l'homme a autorité dans l'espace public, et la femme est plus ou moins confinée à la sphère domestique. Mais à l'intérieur de cette sphère domestique, nombre de questions sont du ressort de la femme, dont les décisions et manières de faire s'imposent aux hommes de la maison ${ }^{19}$.

\footnotetext{
${ }^{17}$ Ibid., p. 274.

${ }^{18}$ Ibid., p. 274-275.

${ }^{19}$ Un relatif confinement était aussi, au départ, une mesure de prudence et de protection. Par ailleurs, il a été montré que dans de nombreuses sociétés rurales, le sphère domestique s'avère au bout du compte plus
} 
La question n'est nullement de magnifier le passé par rapport au présent ou vice versa. Il s'agit de mieux comprendre les difficultés spécifiques auxquelles nous devons aujourd'hui faire face, en les comparant aux difficultés auxquelles les sociétés anciennes étaient ellesmêmes confrontées. Les sociétés traditionnelles ont du mal à reconnaître les différences purement individuelles, dans la mesure où, en leur sein, un être est d'abord défini par les groupes auxquels il appartient, les structures dans lesquelles il est inscrit. Les sociétés modernes sont mieux à même de répondre au désir de la personne d'être reconnue pour ellemême $^{20}$. Elles n'en ont pas moins d'autres problèmes. Pour chacun d'entre nous, la reconnaissance des différences individuelles va de pair avec des évaluations personnelles; mais ces évaluations ne posent pas de difficultés sur le plan social dans la mesure où, précisément, elles demeurent personnelles. Cependant, l'individu découvre qu'être reconnu en tant que soi-même ne suffit pas ; ou plutôt, il découvre que des appartenances collectives font partie de son être : sexe, origine, religion, etc. participent à ce qu'il est. En même temps, la reconnaissance d'une appartenance collective ne peut plus être seulement une affaire individuelle, elle implique nécessairement la collectivité en tant que telle; et si cette reconnaissance ne peut éviter d'impliquer des valeurs, ces valeurs sont également collectives. Or, les valeurs induisent des hiérarchies, de sorte que, inévitablement, la demande de reconnaissance va entrer en tension avec l'exigence d'égalité.

Plutôt que de raisonner dans le vide, prenons encore une fois des exemples.

Vis-à-vis des religions : la solution «laïque ${ }^{21} »$ consiste à considérer l'appartenance religieuse comme une affaire purement privée. Cette solution ne va pas sans violence : elle ne rend pas justice à ce qu'est véritablement une religion. Cependant, une reconnaissance publique pose de redoutables problèmes eu égard à l'exigence d'égalité. Que peut signifier, en effet, un traitement égal de l'appartenance et de la non-appartenance religieuse ? Et un traitement égal de religions différentes par leur nature, leur contenu, leur histoire, le nombre et l'engagement de leurs adeptes, etc. ? Les principes sont utiles pour ordonner la pensée,

importante que la sphère publique, et que les femmes accordent aux hommes un pouvoir symbolique en échange d'un pouvoir réel (voir par exemple : Susan Carol Rogers, «Female form of power and the myth of male dominance: a model of female/male interaction in peasant society », American Ethnologist, vol. 2, $\mathrm{n}^{\circ} 4,1975$, p. 727-756). En revanche, la condition de la femme a connu un moment de forte dégradation avec l'avènement de la société industrielle.

${ }^{20}$ Un désir que, par ailleurs, elles contribuent elles-mêmes à engendrer. Émile Durkheim a mis en lumière ce paradoxe de la situation moderne, où les individus ne mesurent pas que leur revendication d'autonomie par rapport à la société est, pour une large part, un produit de l'organisation sociale elle-même (marquée, en particulier, par une extrême division du travail qui, tout en accentuant la dépendance de chacun vis-à-vis des autres, rend cette dépendance moins sensible qu'en des temps où la collaboration entre individus nécessitait une entente directe entre eux).

${ }^{21} \mathrm{Il}$ y aurait beaucoup à dire sur l'emploi du terme « laïque » dans ce contexte. L'adjectif grec laikos est dérivé du substantif laos, qui désignait le peuple assemblé, et a été repris dans le Nouveau Testament pour désigner le peuple de Dieu. Le laïc, au Moyen Âge, n'était pas hors de la religion, il n'était simplement pas un clerc. 
mais deviennent nocifs quand on prétend tout régler à partir d'eux, sans égards pour la singularité des situations. La seule voie sensée, sur ce terrain, est celle des accommodements raisonnables ${ }^{22}$. (Faute de quoi, les différences substantielles que les principes ont prétendu ignorer font retour sur la scène sociale sous la forme de phénomènes de ségrégation : l'égalité de principe produit une séparation de fait.)

\section{Retour sur la différence hommelfemme}

Nous avons dit de la différence entre hommes et femmes qu'il ne s'agissait pas d'une différence parmi d'autres mais, pour les êtres humains, d'une différence fondatrice, de l'archétype des différences. Ce caractère fondamental explique à la fois sa résistance - elle est toujours un élément essentiel de notre identité, mentionné sur les documents officiels -, et les attaques dont la reconnaissance sociale de la différence des sexes est l'objet. Ces attaques ont plusieurs sources. L'une d'elles, très importante bien que rarement mentionnée, est l'idéologie libérale, et l'extension du modèle économique qui en résulte, englobant progressivement tous les aspects de la vie humaine. Karl Polanyi a montré que toutes les anciennes civilisations, empires y compris, s'étaient entendues à mettre des bornes aux activités commerciales et à la logique économique; et que ce qui caractérise notre époque n'est rien d'autre que l'effacement de ces bornes, d'où résulte un désenclavement (disembedding) de l'économie, qui avait jadis une place limitée au sein de la culture et qui tend aujourd'hui à tout englober (ce qu'on persiste à appeler la culture n'étant plus qu'un secteur économique parmi d'autres). En s'interrogeant sur les conditions qui ont rendu possible un tel événement, allant de pair avec la mort progressive de toutes les cultures, Ivan Illich a souligné l'importance, à ses yeux cruciale, du passage d'un monde "genré », peuplé d'hommes et de femmes, à un monde « sexué », peuplé d'être humains affectés, secondairement, d'un sexe masculin ou féminin ${ }^{23}$. Dès lors qu'on reconnaît à la différence homme/femme une dimension irréductible, alors d'autres limitations peuvent être admises - ainsi le confinement de la logique économique à certains types d'activité. Dans le cas contraire, toute limite est susceptible d'être mise en question et répudiée comme arbitraire. Est alors enclenchée une dynamique de dédifférenciation générale, de «pourtoussisme», et d'arasement de toutes les distinctions

\footnotetext{
${ }^{22}$ C'est un tel accommodement qui a été trouvé vis-à-vis de l'Italie par l'Union européenne qui, saisie en 2006 par une plaignante, avait en première instance proscrit la présence de crucifix dans les salles de classe de l'école publique, comme attentatoire à la liberté de conscience. La Cour européenne des droits de l'homme a infirmé cette décision en mars 2011. Qu'il nous soit permis de considérer que la présence maintenue des crucifix dans les écoles italiennes, en conformité avec l'histoire du pays, est plus favorable, à terme, que n'aurait été leur retrait imposé, à la coexistence pacifique dans ce pays des catholiques avec ceux qui appartiennent à une autre religion ou n'en confessent aucune.

La notion d'«accommodement raisonnable » est très utilisée au Canada, y compris dans des cas où les accommodements trouvés semblent assez déraisonnables.

${ }^{23}$ Voir Le Genre vernaculaire [Gender, 1982], trad. de l'anglais par Maud Sissung, in Euvres complètes, 2 vol., Fayard, vol. 2, 2005. Dans cet ouvrage, l'usage que fait Illich des termes de «genre » et de «sexe » est très différent de celui qui a cours aujourd'hui.
} 
patiemment édifiées par les cultures humaines pour structurer l'espace, le temps et leurs usages ; alors tout peut se transformer en tout, tout peut être livré aux entreprises techniques et aux échanges économiques. Autrement dit, Illich établit un lien entre des maux contemporains que beaucoup s'accordent à fustiger, et une dynamique d'indifférenciation entre les hommes et les femmes que les mêmes s'accordent souvent à considérer comme éminemment positive, et insuffisamment accomplie.

À la dynamique libérale s'allient, pour sinon nier, du moins «secondariser» la différence des sexes, les tendances gnostiques de la modernité. Eric Voegelin est allé jusqu'à écrire: «L'essence de la modernité consiste en un accroissement du gnosticisme ${ }^{24}$.» Le gnosticisme ancien trouvait ce monde-ci très mauvais, et nourrissait l'espoir de se défaire de tout élément charnel. Le gnosticisme moderne trouve également ce monde fort mal fait. Mais son ambition, désormais, n'est pas de s'en extraire, elle est de le rendre bon en le transformant. On ne saurait vraiment comprendre l'activisme technique moderne tant qu'on ne saisit pas la dimension messianique qui l'habite. Et on ne saurait vraiment comprendre le matérialisme moderne, si souvent dénoncé, si on ne mesure pas à quel point ce matérialisme est la contrepartie d'un spiritualisme radical. Il ne faut pas s'y tromper : même si un certain esprit scientifique moderne se revendique comme moniste, ce prétendu monisme est en réalité un dualisme si exacerbé qu'il en perd la faculté de se reconnaître comme tel ${ }^{25}$. Mais il ne s'agit plus, comme dans les temps anciens, d'échapper à la matière par l'esprit, il s'agit de soumettre entièrement la matière à l'esprit. Ernest Renan donnait le mot d'ordre, dans L'Avenir de la science: "Le grand règne de l'esprit ne commencera que quand le monde matériel sera parfaitement soumis à l'homme ${ }^{26}$. On ne parle plus d'âme. Cependant, une autre entité métaphysique a pris sa place: une volonté impérieuse, impérialiste, revendicatrice, devant laquelle tout doit plier. De là l'agressivité particulière à l'encontre du donné, de tout donné, de tout ce qui pourrait paraître intangible ou indisponible : le passé, la tradition, la nature. Le passé doit être critiqué, la tradition doit être renversée, la nature doit être maîtrisée et domestiquée. Fatalement, ce mouvement d'émancipation à l'égard du donné en vient à se heurter à un donné fondamental : la différence des sexes. Face à la réquisition générale du monde par la volonté, voilà un obstacle de taille ; peut-être l'obstacle suprême. On connaît l'adage du juriste et philosophe politique Jean-Louis de Lolme qui, pour critiquer les pouvoirs du Parlement britannique qu'il estimait exorbitants, disait que le Parlement pouvait tout faire sauf changer une femme en homme ou un homme en femme ${ }^{27}$ : le transsexualisme était une figure de l'impossible. Au Moyen Âge le diable, malgré les

\footnotetext{
${ }^{24}$ La Nouvelle Science du politique. Une introduction [1952], trad. Sylvie Courtine-Denamy, Seuil, 2000, p. 183.

${ }^{25}$ Celui qui, par exemple, affirme que la pensée est un processus purement matériel n'est pas, comme il se revendique lui-même, matérialiste, il est en fait tellement idéaliste qu'il ne s'en aperçoit plus. C'est précisément de cette position idéaliste ignorée qu'il peut déclarer que tout est matière. Descartes, dans son dualisme assumé, était autrement plus conséquent que ceux qui le regardent aujourd'hui avec condescendance.

${ }^{26}$ Paris, Calmann-Lévy, 1890, chap. IV, p. 80.

${ }^{27}$ Constitution de l'Angleterre, ou état du gouvernement anglais comparé avec la forme républicaine et avec les autres monarchies de l'Europe [1771], éd. Jean-Fabien Spitz, Dalloz, 2008.
} 
innombrables pouvoirs qu'on lui prêtait, n'avait pas celui de changer le mâle en femelle, et réciproquement. (Quant à Dieu, il s'est toujours abstenu de procéder à pareille opération.) Pour le petit enfant, la rencontre de la différence sexuelle est une étape cruciale dans la reconnaissance du principe de réalité. La vérité du sexe comme réel, écrit Lacan, c'est qu'il y en a deux - et que chaque être est situé d'un seul côté, et donc délogé de sa complétude imaginaire. Réciproquement, on perçoit l'enjeu sous-jacent à la négation ou à la marginalisation de la différence sexuelle : pouvoir continuer à bercer un fantasme de toutepuissance et de complétude personnelle ${ }^{28}$. Et puisque «l'anatomie, c'est le destin ${ }^{29} »$, puisqu'il n'y a pas de corps humain mais seulement des corps d'hommes et des corps de femmes $^{30}$, il faut pour prétendre à la complétude récuser l'anatomie, soit en la modifiant par des interventions techniques, soit en la déclarant subsidiaire. À un donné, naturel et social, qui nous définit, on entend alors substituer une identité choisie par l'individu. Dans ce refus de l'esprit à se sentir lié par tout donné corporel, dans ce refus de la dimension charnelle de l'âme humaine on reconnaît une inspiration typiquement gnostique. Participe à cette inspiration une forme d'ivresse symbolique, liée à une inversion du rapport entre le réel et le système symbolique de notation qu'on emploie pour l'évoquer : quand le réel en vient à être pensé exclusivement à partir du système symbolique, et que ce système use de signes réputés arbitraires (comme c'est le cas de nos alphabets), alors il est devient concevable de passer de l'arbitraire du signe à l'arbitraire de ce dont il est signe - de la manipulation à volonté des symboles à l'idée que la réalité doit se plier sans résistance à cette même volonté.

Par ailleurs, la prise en compte de la différence homme/femme se heurte aujourd'hui à une grave accusation: être un instrument de perpétuation de la domination masculine. L'horreur qu'inspire l'idée de domination - qui, entre humains, n'a généralement rien de sympathique (même s'il faudrait distinguer entre de nombreux modes de domination) s'étend à celle de hiérarchie (même si, nous l'avons dit, hiérarchie n'implique pas domination au sens de pouvoir). Cependant, une fois encore, Louis Dumont nous met en alerte, lorsqu'il affirme : «Il y a deux voies pour reconnaître l'Autre : la hiérarchie et le conflit ${ }^{31}$. » Certes, Dumont n'est pas un oracle ; cependant, quand bien même sa proposition serait discutable, elle a le mérite de signaler une difficulté, là où une certaine naïveté contemporaine pense qu'il

\footnotetext{
${ }^{28}$ D'où ces paroles de Freud: «Quiconque promettra à l'humanité de la libérer des difficultés du sexe [comprendre ici : de la différence des sexes] sera acclamé comme un héros, quelles que soient les absurdités qu'il puisse proférer» (lettre du 17 mai 1914, in The complete correspondence of Sigmund Freud and Ernest Jones, 1908-1939, Cambridge (Mass.), Belknap Press of Harvard University Press, 1993).

${ }^{29}$ Sigmund Freud, « La disparition du complexe d'Edipe » [1923], trad. Denise Berger, in La Vie sexuelle, Paris, P.U.F., 1969, p. 121.

${ }^{30}$ L'invocation des quelques cas naturels ambigus n'est pas une objection solide : c'est comme si l'on devait se refuser à dire que les êtres humains ont deux bras et deux jambes au nom du fait qu'il naît des enfants sans membres ou avec des membres supplémentaires. Au passage, le fait que ce soit précisément la différence sexuelle qu'on cherche à lisser, en arguant de cas exceptionnels, est significatif du statut singulier de cette différence.

${ }^{31}$ Essais sur l'individualisme, op. cit., p. 298.
} 
suffit de défaire les hiérarchies pour que règne la paix et l'harmonie. Un exemple anecdotique permet d'illustrer le propos de Dumont. Dans les albums de Tintin les deux policiers, Dupond et Dupont, rivalisent d'amabilité au moment de franchir une porte : Après vous, Je vous en prie, Je n'en ferai rien... Finalement, ils se décident en même temps à passer la porte et entrent en collision. Faute de hiérarchie établie dans l'ordre de passage, il y a conflit. Autre exemple : il était d'usage en anglais, pour désigner un être humain générique, d'employer le pronom he (de même qu'en français, en pareil cas, on a recours au $i l$ ). Les féministes se sont élevées contre ce recours au pronom masculin et, pour y remédier, plusieurs propositions ont été avancées : l'emploi de s/he (lourd et imprononçable), de he or she ou de she or he (encore plus lourds). Finalement, la solution retenue pas de nombreux éditeurs a été d'opter pour she - cette préséance étant accordée au pronom féminin à titre de compensation, en considération des nombreux siècles d'emploi du $h e^{32}$. On voit, sur ce cas très simple, que la rigoureuse égalité est impraticable, et que le seul moyen d'échapper à de perpétuelles controverses est de fixer une hiérarchie, qu'on s'est ici contenté d'inverser par rapport à l'usage antérieur.

Le recours aux hiérarchies est si important (comment, par exemple, pourrait-on classer les mots dans un dictionnaire sans ordre entre les lettres) qu'il est essentiel d'éviter la confusion avec les rapports de domination - même si, dans le domaine humain, le glissement des unes aux autres est toujours susceptible de se produire. Un moyen de neutraliser, autant que faire se peut, un tel glissement, est d'inverser les hiérarchies selon les contextes : dans un registre, A passe avant B, mais, dans un autre registre, B passe avant A.
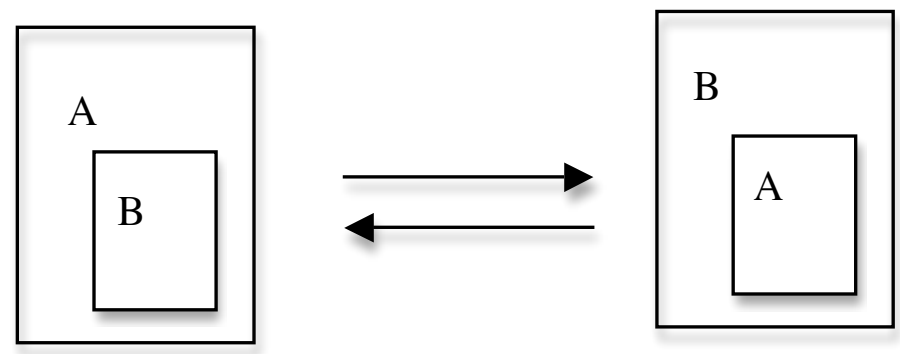

Notons, néanmoins, que cet équilibre est instable, dans la mesure où le registre où $\mathrm{A}$ passe avant $B$ risque toujours de paraître plus important que le registre où $B$ passe avant $A$ (ou inversement), ce qui nous reconduit à la figure de la hiérarchie enchevêtrée déjà évoquée, où la hiérarchie ne s'inverse que dans un domaine subordonné. Par ailleurs, de telles figures risquent toujours de paraître trop rigides à un individu qui entend décider par lui-même de la place qu'il occupe dans la société : ainsi, même si la reconnaissance de la différence sexuelle allait de pair avec un parfait équilibre, un individu pourrait toujours se trouver insatisfait des modalités selon laquelle cette différence serait prise en compte. Pour certaines personnes, c'est au nom du droit à la différence que toutes les options possibles et imaginables doivent

\footnotetext{
${ }^{32}$ Cette règle est en usage dans la plupart des revues académiques aux États-Unis et en Australie. Elle stipule également que lorsqu'un texte met en scène deux personnes génériques, dont l'une s'adresse à une autre, la première doit être désignée par she, la seconde par he (pour contrer le stéréotype d'un féminin qui serait « second » par rapport au masculin).
} 
être reconnues comme normales. Pour d'autres personnes, c'est au nom du fait que les différences, à bien y regarder, n'en sont pas vraiment, ou que les frontières ne passent pas où on le croyait. Le moment serait venu, par exemple, de reconnaître que la frontière entre le masculin et le féminin ne passe pas entre les hommes et les femmes, mais à l'intérieur de chacun de nous $^{33}$. Il y a, certes, cette persistante et irritante dissymétrie entre les femmes et les hommes, qui veut que ce soient les premières qui conçoivent les enfants, mais les travaux sur la possibilité de grossesse masculine, ou la mise au point de l'utérus artificiel, en viendront peut-être à bout. Alors la société sera délivrée de la distinction archaïque entre hommes et femmes, elle connaîtra seulement des êtres humains, tous bisexuels, enfin libres d'exprimer pleinement leurs diverses potentialités. Le point de départ du raisonnement est exact : il y a du masculin et du féminin en chacun de nous. Mais encore faut-il, pour appréhender cette différence à l'intérieur de soi, qu'elle commence par être reconnue au dehors - qu'elle commence par distinguer des individus : on se demande comme les êtres humains auraient l'idée d'identifier en eux du masculin et du féminin s'il n'y avait au dehors des hommes et des femmes ${ }^{34}$. Sans organisation externe, il n'y a qu'un magma interne. Prenons une image : celle de l'oiseau évoqué par Kant ${ }^{35}$ - la colombe qui, sentant son vol freiné par la résistance de l'air, imagine qu'elle volerait mieux dans le vide. Mais dans le vide, la colombe ne pourrait pas décoller. De même, ceux qui imaginent que la reconnaissance sociale de la différence entre hommes et femmes est une entrave à la liberté et à l'expression individuelle, ne se rendent pas compte que cette différence est un des fondements de l'individu dont ils souhaitent l'apothéose.

$* * *$

\footnotetext{
${ }^{33}$ Voir Élisabeth Badinter, L'Un est l'autre, Odile Jacob, 1986. « La ressemblance [entre hommes et femmes] est moins l'effet d'une neutralisation du sexuel que de la bisexualité commune aux deux sexes » (p. 273). Il est à noter que l'homonymie entre «l'un est l'autre » et «l'un hait l'autre » n'est peut-être pas qu'un vain jeu de mots de notre part : sans l'école de l'altérité qu'est la réalité et la symbolique de la différence des sexes, plus rien ne vient faire obstacle aux identifications imaginaires, aux emballements mimétiques et aux conflits sans merci qui en résultent.

${ }^{34}$ Des manuels scolaires enseignent désormais que l'identité sexuelle ne se réduit pas à un donné biologique, mais qu'elle se construit tout au long de la vie. L'ennuyeux n'est pas l'assertion en elle-même, qui est vraie, mais la façon dont elle sera généralement interprétée dans le contexte actuel qui ne reconnaît que deux autorités, la nature et l'individu; de sorte que l'identité sexuelle, dès lors que se trouve affirmée en elle une dimension construite, sera réputée être à la disposition de l'individu - comme si on pouvait devenir un être humain accompli par ses propres moyens. En réalité, quand la société refuse de transmettre aux nouveaux venus les modèles identificatoires à partir desquels ils pourront accéder à une certaine autonomie, et aller vers l'autre, le vide est rempli par les images d'une extrême pauvreté proposées par le marché, la mode, des sous-cultures trash - dans la plupart des cas plus sexistes, remarquons-le, que ne l'était la culture classique. Plus les adultes refusent d'authentifier la différence des sexes, plus les enfants et les adolescents ont tendance à outrer cette différence pour se fabriquer une identité. (Voir, par exemple, Dominique Pasquier, Cultures lycéennes, Autrement, 2005, p. $80 s q$.)

${ }^{35}$ Critique de la raison pure, Introduction, III [2 2 édition, 1787].
} 
Pour concilier, autant qu'il est possible, les demandes de reconnaissance avec l'exigence concomitante d'égalité, les revendications de liberté dans les choix personnels et la nécessité de règles communes pour qu'une société soit viable, il n'existe pas de solution générale. Tocqueville, parlant des philosophes du XVIII siècle, remarquait : "Tous pensent qu'il convient de substituer des règles simples et élémentaires, puisées dans la raison et la loi naturelle, aux coutumes compliquées et traditionnelles qui régissent la société de leur temps. En y regardant bien, l'on verra que ce qu'on pourrait appeler la philosophie politique du XVIII $I^{\mathrm{e}}$ siècle consiste à proprement parler dans cette seule notion-lăa ${ }^{36}$.» Néanmoins, on doit bien constater à la longue qu'un équilibre est à trouver entre, d'un côté, l'universalisme, de l'autre le fait que l'on n'accède pas à l'universel en niant les déterminations particulières, d'un côté la fidélité à des principes, de l'autre la sensibilité aux cas concrets (ne serait-ce que parce que les principes, laissés à eux-mêmes, entrent en conflit les uns avec les autres). Comme un marcheur doit savoir, dans une contrée inconnue, se confier non seulement à sa boussole, mais aussi au relief, à la végétation et aux possibilités de se frayer un chemin, nous devons savoir, pour répondre aux questions inédites que le monde nous pose, composer avec le donné, naturel et historique, les traditions et les héritages, l'expérience, le concret, la substance et la résistance du réel qui est, d'une certaine manière, la première caractéristique de ce réel, ce qui fait que le réel est réel et que, même s'il lui arrive de nous tourmenter, nous pouvons compter sur lui.

La reconnaissance des différences dans l'égalité : nous devons être conscients qu'il ne peut s'agir là que d'un idéal qui, en tant que tel, n'est pas à portée de main, et est sans doute impossible à atteindre. Un idéal qui n'a peut-être pas de sens : car que peut signifier la reconnaissance d'une différence, sans une influence sur le comportement ? Et comment un comportement ainsi modifié peut-il encore être qualifié d' «égal»? Saint Augustin définit la vertu comme un «ordo amoris », un ordre au sein duquel chaque être, chaque objet reçoit le genre et le degré d'amour qui lui est approprié ${ }^{37}$. Mais comment établir ce qui est approprié ? Peut-être que ce qui devrait être également accordé à chacun, à chaque situation, ce serait l'attention - l'attention dont procède le comportement juste. Mais, comme l'a remarqué Simone Weil, «il y a quelque chose dans notre âme qui répugne à la véritable attention beaucoup plus violemment que la chair ne répugne à la fatigue ${ }^{38} \gg$. C'est pourquoi, faute de mieux, nous réclamons l'égalité : comme une façon de composer avec nos faiblesses, comme une sorte de suspension de jugement, parce que nous n'arrivons pas à juger justement. Mais le mieux serait d'être attentif, autant qu'il nous soit donné de l'être.

\footnotetext{
${ }^{36}$ L'Ancien Régime et la Révolution [1856], Flammarion, coll.GF, 1988, p. 230.

${ }^{37}$ La Cité de Dieu, XV, 22.

38 « Réflexion sur le bon usage des études scolaires en vue de l'amour de Dieu », in Euvres complètes, IV, 1, Gallimard, 2008, p. 259.
} 